\title{
Medikationsfehler mit Morphin - eine systematische Analyse
}

Olga Frank ${ }^{a}$, Carmen Kerker-Specker ${ }^{b}$, Chantal Zimmermann $^{c}$, David Schwappach ${ }^{d}$

${ }^{a}$ Dr., Leitung Projekte, Produkte, Dienstleistungen, Patientensicherheit Schweiz

${ }^{b} \mathrm{MScN}$, Projektmanagerin, Patientensicherheit Schweiz

c M.A., Projektmanagerin, Patientensicherheit Schweiz

${ }^{d}$ Prof. Dr., MPH, Wissenschaftlicher Leiter, Patientensicherheit Schweiz

\section{Die Stiftung Patientensicherheit Schweiz hat alle Fehlermeldungen in der CIRRNET-} Datenbank, welche die Thematik Morphin aufgreifen, systematisch analysiert und spezifische Problemfelder identifiziert. Am häufigsten kommen Medikationsfehler mit Morphin in der Phase der Ausgabe bzw. der Vorbereitung vor. Neben Berechnungsfehlern sind es auch Fehler bei der Programmierung von Infusomaten/ Perfusoren oder Verwechslungen von Morphin und Pethidin.

\section{Einleitung}

Medikationsfehler gehören zu den häufigsten Fehlertypen sowohl im ambulanten als auch im stationären Bereich. Daher stellen sie für die Patientensicherheit ein grosses Risiko dar. Es kann beispielsweise zu Verzögerungen im Heilungsprozess kommen, zusätzlichen Behandlungskosten, unerwünschten Nebenwirkungen oder bleibenden Gesundheitsschäden. Um Medikationsfehler zu vermeiden, sind Kenntnisse über Risikofaktoren und deren Ursachen wichtig. Medikamente, die häufig mit Medikationsfehlern in Zusammenhang stehen und mit schweren Folgen für den Patienten verbunden sein können, sind dabei von besonderem Interesse. Zu diesen Medikamenten zählt Morphin, welches das Institute for Safe Medication Practices (ISMP) [1] auf der Liste der Hochrisikomedikamente für den akutstationären Bereich führt.

Patientensicherheit Schweiz hat aufgrund der hohen Relevanz der Medikationsfehler mit Morphin alle Fehlermeldungen in der CIRRNET*-Datenbank mit einer beschriebenen Morphinproblematik systematisch analysiert. Zudem wurden spezifische Problemfelder entlang der einzelnen Schritte im Medikationsprozess identifiziert. Eine solche Auswertung der CIRRNETMeldungen ist eine Möglichkeit, mehr über Medikationsfehler bei der Verwendung von Morphin zu erfahren. Da die Weiterleitung lokaler Fehlermeldungen an die CIRRNET-Datenbank selektioniert durch die CIRRNET-Teilnehmer erfolgt und in Fehlermeldesystemen nicht jedes sicherheitsrelevante Ereignis gemeldet wird, sind die nachstehenden Ergebnisse nicht repräsentativ für Medikationsfehler mit Morphin. Die angewandte Methode zeigt jedoch, dass die Identifikation von spezifischen Problemfeldern möglich ist, die sich wiederum für die Entwicklung von Verbesserungsmassnahmen in den Gesundheitsinstitutionen eignen.

\section{Methode}

Die CIRRNET-Datenbank wurde mittels einer Freitextsuche mit festgelegten Stichworten (Morph, BTM, Capros, Kapanol, MST, MSR, MSI, M-STADA, M-long, M-beta, M-retard, Schmerzpflaster, Sevre) auf relevante Fehlermeldungen für den Zeitraum vom 25.9.2006 bis 31.12.2014 durchsucht. Die Fehlermeldungen wurden von zwei Personen mit Fachexpertise nach den fünf Phasen des Medikationsprozesses («Verschreibung/Verordnung", «Dokumentation", "Ausgabe/Vorbereitung», «Verabreichung», «Monitoring/laufender Betrieb») [2] klassifiziert (Abb. 1) und spezifische Problemfelder identifiziert. Die Übereinstimmung der Kategorisierung nach den Phasen des Medikationsprozesses beider Untersucherinnen betrug 87,5\% aller Meldungen.

\section{Resultate}

Insgesamt konnten 64 Fehlermeldungen in die Analyse eingeschlossen werden. In Box 1 sind zwei originale CIRRNET-Meldungen beispielhaft dargestellt. Die Analyse zeigt, dass sich 14,1\% der CIRRNET-Meldungen auf den Prozessschritt der ärztlichen Verschreibung/Ver- 


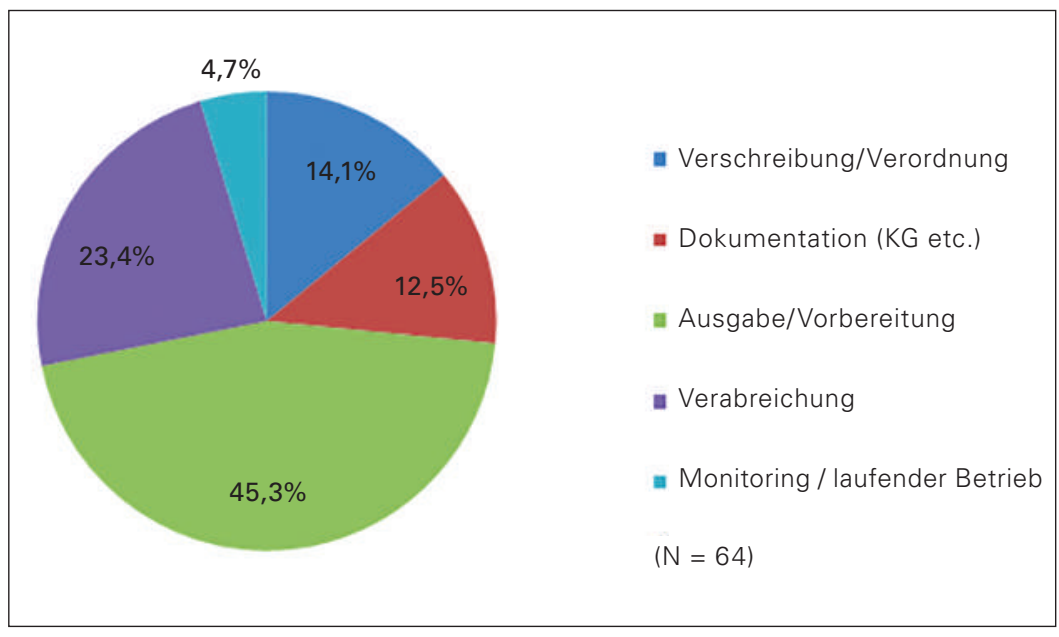

Abbildung 1: Verteilung der eingeschlossenen CIRRNET-Meldungen auf die Phasen des Medikationsprozesses.

ordnung beziehen. Sie beschreiben ausschliesslich unvollständig und/oder unverständlich dokumentierte Verordnungen von Morphin, wie fehlende Angaben zur Dosierung oder Verabreichungsform.

12,5\% der Meldungen betreffen die Dokumentation von Morphin. Als Problemfelder werden Übertragungsfehler, unberücksichtigte Allergien und fehlerhafte Dokumentation der Galenik von Morphin genannt, wobei Übertragungsfehler am häufigsten beschrieben werden. Ursachen solcher Übertragungsfehler können Übermüdung, Überlastung, Unaufmerksamkeit oder auch Kommunikations- oder Informationsdefizite sein. Knapp die Hälfte aller CIRRNET-Meldungen $(45,3 \%)$ bezieht sich auf die Ausgabe und Vorbereitung von Morphin. Aufgrund der zahlreichen spezifischen Arbeitsschritte während der Vorbereitung eines Medikaments, wie bspw. das Richten von Morphin zur i.v. Applikation,

\section{Box 1: Original CIRRNET-Meldungen}

Original-CIRRNET-Meldung zum Prozessschritt "Verschreibung/Verordnung»:

«Pat. hat Atemnot. Res.-Medi. ist Morphin $1 \mathrm{mg}$. Pflege versucht DA zu erreichen, da die Res.-Verordnung nicht zeigt in welcher Form das Morphin verabreicht werden soll und wie häufig es gegeben werden darf. Da Situation akut, entscheidet sich PP für Morphintropfen und verabreicht der Pat. 4 Tropfen Morphin 2\%.Nach der Verabreichung hat PP nochmals die Dosierung nachgeprüft, da sie sich in Ungewissheit sah bzgl. der Tropfenmenge. DA nochmals versucht zu kontaktieren und ihm dann Situation geschildert. Man hätte Morphin 1 mg i.v. geben können. Pat. soll Kontrolle erhalten, da von der Dosierung her 1-2 Tropfen genügt hätten, jedoch keine Komplikationen zu erwarten seien meint DA hat dann auch die Reserveverordnung angepasst. OA hat noch vorbeigeschaut und sich erkundigt, und die Verordnung nochmals korrekt aufgeschrieben.»

Original-CIRRNET-Meldung zum Prozessschritt "Ausgabe/Vorbereitung»:

"Arzt verordnete auf Morgenvisite bei terminaler Pat. $20 \mathrm{mg}$ Morphin/24 h in $500 \mathrm{ml} \mathrm{RL}$. Pflegende spritzte diese Dosis Morphin in eine Infusion, welche bereits am Vorabend um 21 Uhr angehängt wurde und somit nur noch maximal halb voll war. Am Nachmittag bemerkte Spätdienst dies, da Pat. immer stärker eintrübte, Pupillengrösse und Reaktion verändert waren und grosses Unwohlsein äusserte. Pat. erhielt durch den Fehler die Tagesdosis $(20 \mathrm{mg})$ innerhalb von \pm 6 Stunden.» kann vermutet werden, dass es hier ein grosses Fehlerpotential gibt. Dabei spielen Unterbrechungen des Arbeitsprozesses eine wesentliche Rolle. Auch Berechnungsfehler, die jedoch nicht ausschliesslich auf Morphin zutreffen, sind ein Problemfeld. Berechnungsfehler sind in diesem Fall aber bei einer Überdosierung mit besonderen, im schlimmsten Fall sogar tödlichen Risiken für den Patienten verbunden. Auffällig ist, dass die Fehlermeldungen überwiegend Überdosierungen mit Morphin beschreiben. Unterdosierungen kommen kaum vor. An dieser Stelle ist von Interesse, ob Unterdosierungen tatsächlich nicht vorkommen, oder ob diese von den Fachpersonen als nicht gravierende Medikationsfehler eingestuft und deshalb nicht gemeldet werden.

Die Ergebnisse der Analyse zeigen ausserdem, dass die korrekte Programmierung der Fliessgeschwindigkeit von Perfusoren oder Infusomaten ein Problemfeld darstellt. Jedoch betrifft dieses Problem nicht nur die Verabreichung von Morphin, sondern generell die Schwierigkeit im korrekten Umgang mit Medizintechnik. Besondere Aufmerksamkeit bei der Verwendung von Morphin ist auch der Look-alike-Problematik mit Pethidin-Ampullen zu schenken. Bei vier von fünf Fehlermeldungen, die eine Look-alike-Problematik beschreiben, handelt es sich um eine Verwechslung mit Pethidin.

Auf den Prozessschritt der Verabreichung von Morphin beziehen sich 23,4\% der CIRRNET-Meldungen. Am häufigsten werden Überdosierungen beschrieben und technische Fehlfunktionen von Infusomaten oder Perfusoren. Aber auch Verwechslungen des Applikationsweges kommen vor.

Schliesslich betreffen 4,7\% der CIRRNET-Meldungen das Monitoring / den laufenden Betrieb. Ein interessantes Ergebnis in diesem Prozessschritt ist, dass es auch zu «Fehllieferungen» aus der Apotheke kommt. Beispielsweise kann die Lieferung nicht üblich verwendeter Standardkonzentrationen dazu führen, dass sichere Prozesse nicht mehr verlässlich sind und es zu Fehlern kommt.

\section{Diskussion}

Der Medikationsprozess stellt einen wesentlichen Bestandteil des klinischen Alltags einer Gesundheitseinrichtung dar. Zu den ärztlichen Tätigkeiten im Rahmen des Medikationsprozesses gehört beispielsweise das Verordnen von Medikamenten. Betrachtet man die fünf Phasen des Medikationsprozesses, die dieser Analyse als Klassifikation zugrunde gelegt wurden, wird klar, dass es sich um ein komplexes Konstrukt mit einer Vielzahl von parallel laufenden Prozessen han- 
delt. Die Tatsache, dass am gesamten Medikationsprozess immer verschiedene Berufsgruppen involviert sind, kann die Gefahr für Medikationsfehler drastisch erhöhen. Vorwiegend sind es jedoch Ärzte und Pflegefachpersonen, die am Medikationsprozess beteiligt sind. Diese beiden Berufsgruppen müssen sich bewusst sein, dass sie zwar im Einzelnen die Verantwortung für die sichere Durchführung der ihnen zugeteilten Prozessschritte tragen, dass aber der gesamte Medikationsprozess nur sicher ablaufen kann, wenn sich alle beteiligten Berufsgruppen gemeinsam für die Sicherheit des Patienten engagieren.

Die hier durchgeführte Herangehensweise ist eine Möglichkeit der Analyse von Fehlermeldungen. Sie ermöglicht die Identifikation von Problemfeldern bei der Verwendung von Morphin entlang des Medikationsprozesses. Mit derselben Vorgehensweise können Gesundheitsinstitutionen eigene gezielte Analysen durchführen und Verbesserungsstrategien ableiten. Auf überregionaler Ebene sind die Ergebnisse deshalb interessant, weil sie die Problemvielfalt im Medikationsprozess aufzeigen.

Betrachtet man beispielsweise die Ergebnisse der zwei Prozessschritte «Verschreibung/Verordnung» und «Dokumentation", liegt die Überlegung zur Einführung eines elektronischen Verordnungssystems nahe. Die Effektivität solcher Verordnungssysteme zur Erhöhung der Sicherheit des Medikationsprozesses ist hinreichend untersucht $[3,4]$. Allerdings muss bei der Implementierung eines Verordnungssystems berücksichtigt werden, dass ein erfolgreiches Funktionieren eine starke Team- und Kommunikationskultur in der Institution voraussetzt, und die Mitarbeitenden in die hausinternen Sicherheitsbestrebungen eingebunden werden sollten.

Korrespondenz: Dr. Olga Frank

Stiftung Patientensicherheit Schweiz

Asylstrasse 77

CH-8032 Zürich

Tel. 0432441484

Fax 0432441481

frank[at]patienten

sicherheit.ch reduziert [5]. Zur genannten Look-alike-/Sound-alikeProblematik hat Patientensicherheit Schweiz 2010 einen Quick-Alert veröffentlicht, der praktische Handlungsempfehlungen beinhaltet [6].

Die Analyse von Fehlermeldungen ist aufwendig, aber im Vergleich zu einer reinen Häufigkeitsauszählung ertragreicher und weiterführender. Nur durch eine gezielte Analyse von Fehlermeldungen können Problemfelder transparent gemacht und wertvolle Informationen gewonnen werden, die zur Unterbrechung der Fehlerkette beitragen. Die Ergebnisse müssen selbstverständlich kritisch betrachtet werden. Die 64 eingeschlossenen Fehlermeldungen sind nicht repräsentativ, die Klassifikation nach Prozessschritten subjektiv und die Zuordnung in Problemfelder nicht immer eindeutig. Dennoch bieten die Ergebnisse einen Überblick darüber, welche Fehlermeldungen an die CIRRNETDatenbank weitergeleitet werden und welche Probleme bei der Verwendung von Morphin auftreten.

CIRRNET hat zum Ziel, die Patientensicherheit zu erhöhen. Da Fehlermeldesysteme von verschiedenen Berufsgruppen genutzt werden, möchte Patientensicherheit Schweiz möglichst vielen Fachpersonen das gleiche Know-how zugänglich machen. Vor diesem Hintergrund wird ein ähnlicher Artikel auch in der Zeitschrift Krankenpflege publiziert (Nr.11/2015).

Literatur

1 Institute for Safe Medication Practices (ISMP). List of high-alert medications in acute care settings. www.ismp.org/tools/ highalertmedications.pdf

2 Hicks RW, Cousins DD, Williams RL. Selected medication-error data from USP's MEDMARX program for 2002. Am J Health Syst Pharm. 2004;61(10):993-1000.

3 Kaushal R, Kern L, Barron Y, Quaresimo J, Abramson E. Electronic prescribing improves medication safety in community-based office practices. J Gen Intern Med. 2010;25(6):530-6.

4 Nuckols TK, Smith-Spangler C, Morton SC, Asch SM, Patel VM, Anderson LJ, et al. The effectiveness of computerized order entry at reducing preventable adverse drug events and medication errors in hospital settings: a systematic review and meta-analysis. Systematic Reviews. 2014;3(56).

5 Westbrook JI, Woods A, Rob MI, Dunsmuir WTM, Day RO. Association of Interruptions With an Increased Risk and Severity of Medication Administration Errors. Arch Intern Med. 2010;26;170(8):683-90.

6 Stiftung Patientensicherheit Schweiz. Quick-Alert Nr. 14. «soundalike \& look-alike» - Verwechslungsproblematik bei Arzneimitteln, Fokus: Ampullen. www.patientensicherheit.ch/de/ publikationen/Quick-Alerts.html. 